\title{
Pre Surgical Clinical and Echo Assessment of Tetralogy of Fallot
}

\author{
S Khatri, S Shrivastava \\ Director \& Head of Department, Pediatric and Congenital Heart Diseases, \\ Okhla Road, New Delhi, India.
}

(Cardiovasc.j. 2010; 2(2) : 128-135)

Tetralogy of Fallot (TOF) constitutes the most common cause of cyanotic heart disease beyond infancy. In the current era TOF is almost uniformly amenable to surgical repair with good long-term outcomes. The clinical spectrum encountered in TOF is diverse. Symptoms can range from no cyanosis, in the setting of modest Pulmonic stenosis, to profound cyanosis resulting from severe Pulmonic stenosis, pulmonary artery hypoplasia, and resultant right-to-left ventricular level shunting. The evaluation of this diverse anatomy is critical in the preoperative evaluation of a TOF patient to prevent on table surprises to the surgeon.

\section{Clinical Assessment:}

Complete clinical evaluation is important to assess the severity of TOF, look for any syndromes $(7.2 \%)^{1-2}$ and rule out any associated lesion. In all patients clinical assessment includes: weight, age, history of spells, dysmorphic features, degree of cyanosis (saturation), and intensity right ventricular outflow tract and collateral murmur.

Preoperative investigations include: Complete haemogram (hematocrit), Chest $\mathrm{x}$ ray (PA and Lateral), ECG, Septic Screening, Coagulation profile, Renal Function Test, Liver Function Test, Abdominal ultra sound, Cranial U.S. (Infants), G6PD \& Sickle cell Screening (endemic areas).

In severely polycythaemic patient's phlebotomy is needed before surgery

- If $\mathrm{Hb}>20 \mathrm{gm} / \mathrm{dl} \& \mathrm{PCV}>65 \%$, or Symptoms. of Polycythemia present

- Amount of blood :

Observed PCV - Desired PCV x Wt. x Blood Vol. /Kg Observed PCV

Amount of blood to be withdrawn slowly under cardiac monitoring, same volume replaced with normal saline or FFP.
Echo Assessment in Tetralogy of Fallot (TOF)Echocardiography is the most important non invasive imaging modality to delineate the complete pathological anatomy in patients of TOF, this is essential to plan management. While performing Echocardiographic evaluation in TOF, these following steps are essential so that important variations in the morphological abnormalities encountered in Tetralogy of Fallot are not missed and the information obtained is equivalent to that obtained by angiography. ${ }^{3}$

1. Define the atrial situs and the position of the heart in the thorax.

2. Define the atrioventricular connection and the topology (looping - d loop or 1 loop)

3. Define the ventricular arterial connection.

4. Define the pathological anatomy.

a) Ventricular septal defect (VSD) - Site, size and number of ventricular septal defects

b) Aortic override and its degree, presence or absence of aortomitral continuity.

c) Pulmonary stenosis - site (subvalvar, valvar, supravalvar or combinations), presence or absence of infundibular hypoplasia, if present its severity.$^{4}$

d) Pulmonary arteries - confluence, size, stenosis

e) Aortic arch ( right or left)

f) Aortopulmonary collaterals

g) Coronary artery anomalies

5. Measurements: The following echocardiographic measurements are made in all cases.
a) Pulmonary annulus
b) Pulmonary trunk
c) Right pulmonary artery at origin and hilum just before its branching
d) Left pulmonary artery at origin and at hilum just before its branching

Address for Correspondence: Savitri Shrivastava, Director \& Head of Department, Pediatric and Congenital Heart Diseases, Okhla Road, New Delhi, India. 


\section{Ventricular septal defect:}

The ventricular septal defect in tetralogy of Fallot is by definition perimembranous, seen in $75 \%$ cases (Fig 1a, 1b). This is well visualized in the subcostal sagittal and coronal views which show the tricuspid-aortic-mitral continuity. The parasternal short axis view at the level of the aortic valve shows that the ventricular septal defect extends from the area of the tricuspid valve anteriorly to the area of the muscular septum. In about $20 \%$ of cases a muscle bar is present in the posterior inferior margin of the defect and this separates the tricuspid valve from the aortic valve. Echocardiographically this feature is well seen in the subcostal coronal view and in parasternal short axis views. The third and much rarer variety of ventricular septal defect seen in tetralogy of Fallot is the juxtaarterial or doubly committed ventricular septal defect (3\%) best visualised in the subcostal para coronal and parasteranal short axis views (Fig. 2a). ${ }^{6}$
Multiple ventricular septal defects: Additional ventricular septal defects can be best visualized in the subcostal sagittal or the apical 4 chamber views (fig3). Use of the zoom features and low scale of color flow mapping to scan the entire part of the interventricular septum in more detail should be used.

\section{Pulmonary Stenosis:}

The hallmark in tetralogy of Fallot is infudibular stenosis with variable degree of valvar stenosis. This is best seen in the subcostal sagittal, coronal and the parasternal short axis views ${ }^{4-6}$. Valvar pulmonary stenosis is almost always present in tetralogy of Fallot and is demonstrable echocardiographically as doming of the pulmonary valve. The pulmonary annulus in tetralogy of Fallot is almost always hypoplastic and best measured in parasternal short axis views or inferiorly tilted long axis view (Fig. 4).
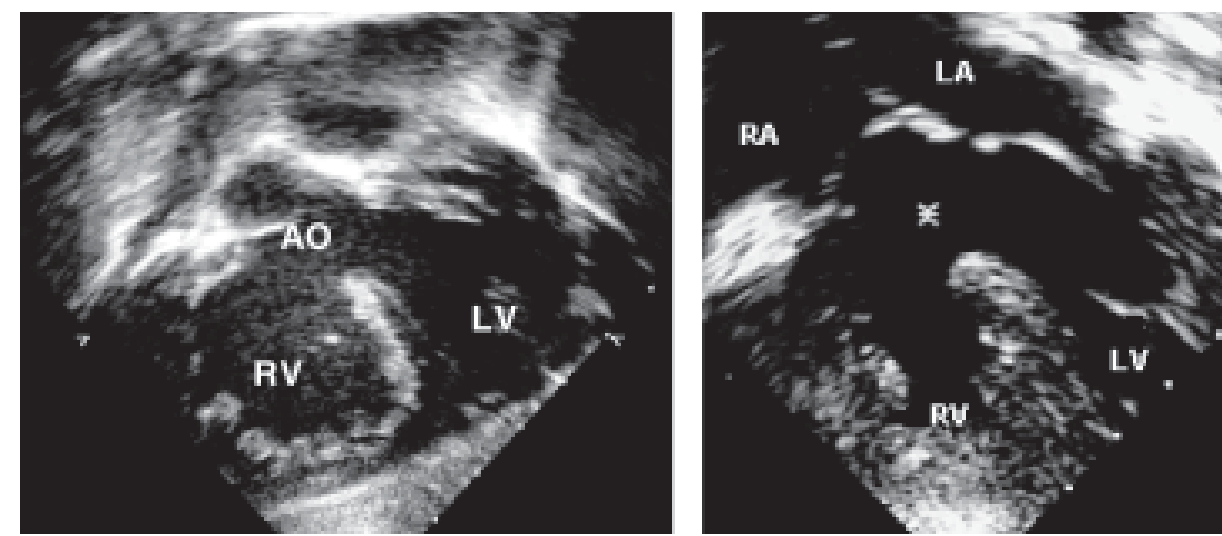

Fig 1: Modified subcostal view showing perimembranous VSD (1A) and perimembranous VSD with inlet extension (1B).
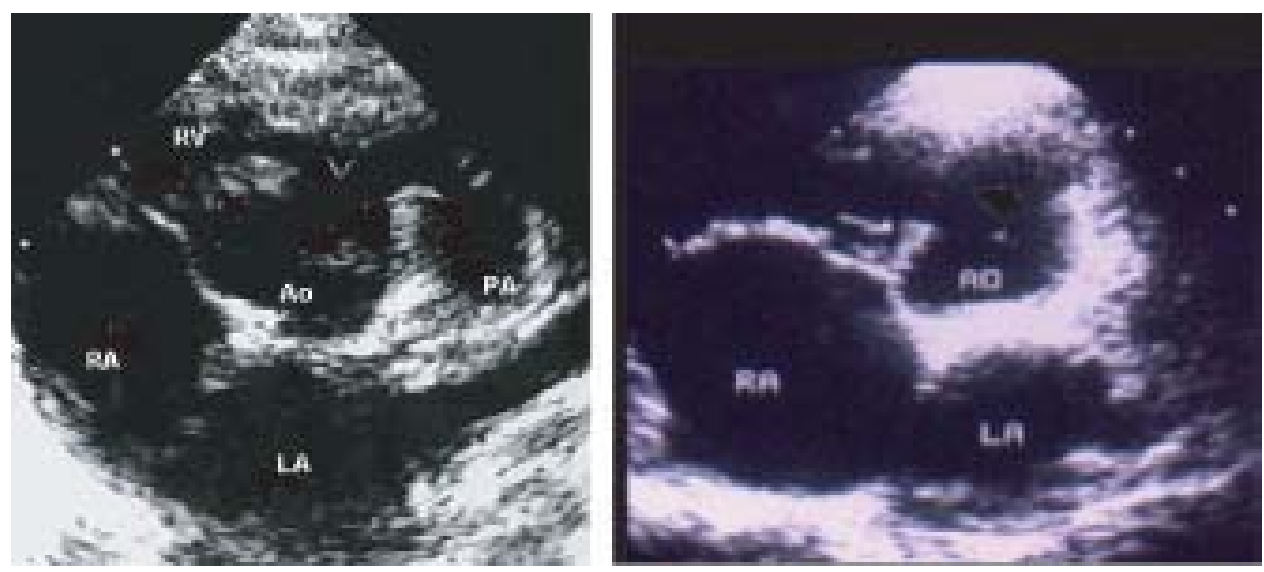

Fig.-2: Parasternal short axis view showing doubly committed VSD (2A) and outlet muscular VSD(2B). 


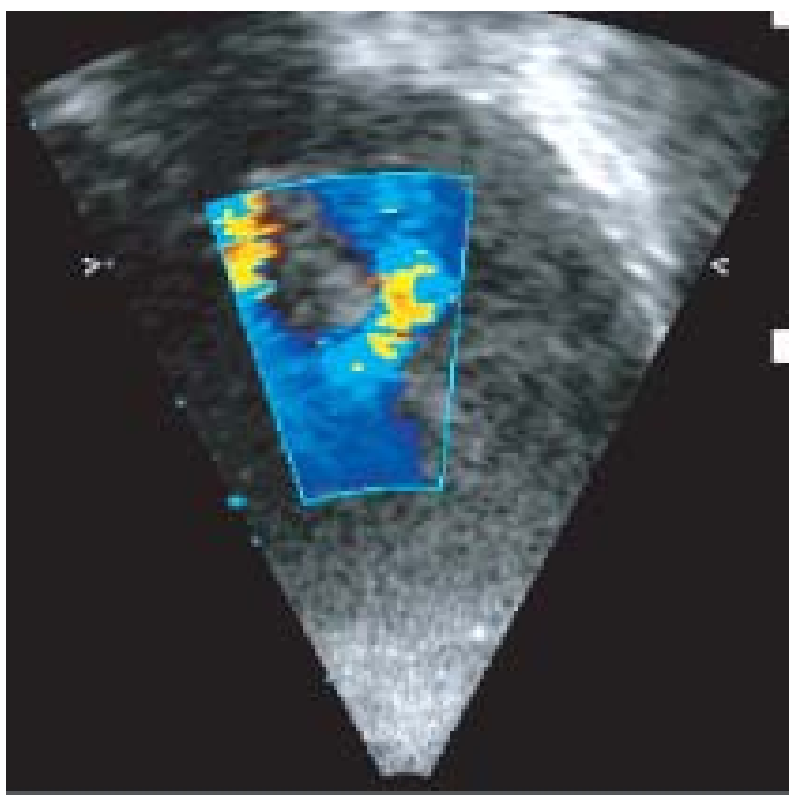

Fig.-3: Modified subcostal view axis view showing additional midmuscular VSD.
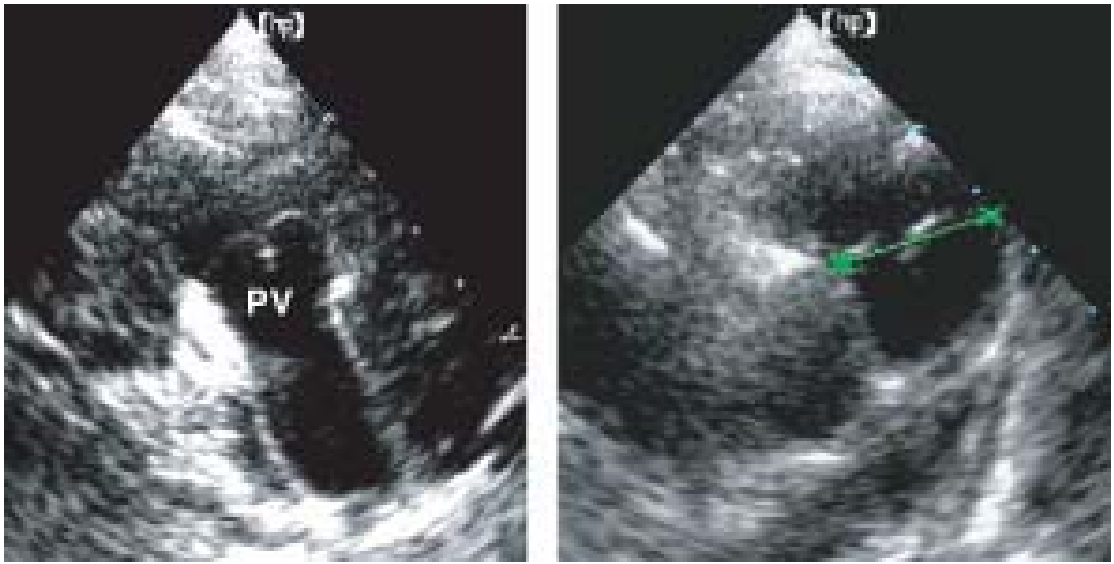

Fig.-4: Modified Parasternal short axis view showing pulmonary annulus.

\section{Tetralogy of Fallot with absent pulmonary valve:}

In this rare variant of TOF the pulmonary valve is absent and replaced by nubs of fibrous tissue, which is best seen in short axis view as bright Echoes. This is associated with significant pulmonary regurgitation clearly seen on color flow mapping. The main/right/left proximal pulmonary arteries may be dilated to variable degrees. The right ventricular obstruction is usually at the level of pulmonary annulus; associated infundibular stenosis may some times be present.

\section{The Pulmonary Arteries:}

Echocardiographically the pulmonary arteries need to be visualized in multiple views. The subcostal coronal view shows the entire length of the right pulmonary artery from its origin to its branching at the hilum. The parasternal short axis view shows the pulmonary confluence, trunk and the origins of the right and left pulmonary arteries and a considerable length of the right pulmonary artery (fig $5 \mathrm{a}, \mathrm{b}$ ). The left pulmonary artery may be profiled in its entire length from the high left parasternal view and the suprasternal oblique long axis view. The suprasternal short axis view opens the entire length of the right pulmonary artery and the left pulmonary artery with anterior tilt (fig6). The pulmonary artery confluences, discrete stenosis, diffuse hypoplasia and supra valvular stenosis should all be looked for in these views. ${ }^{7}$ 


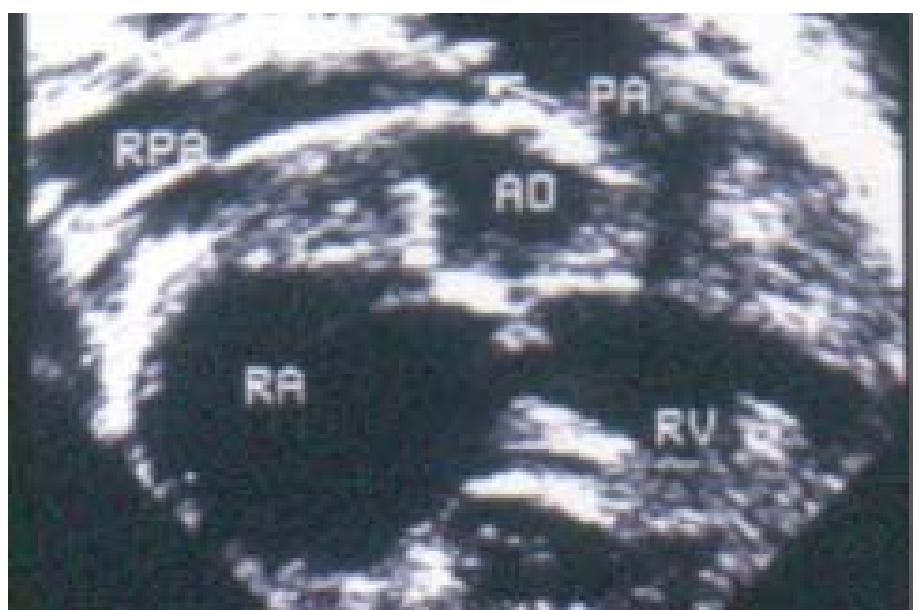

Fig - 5A

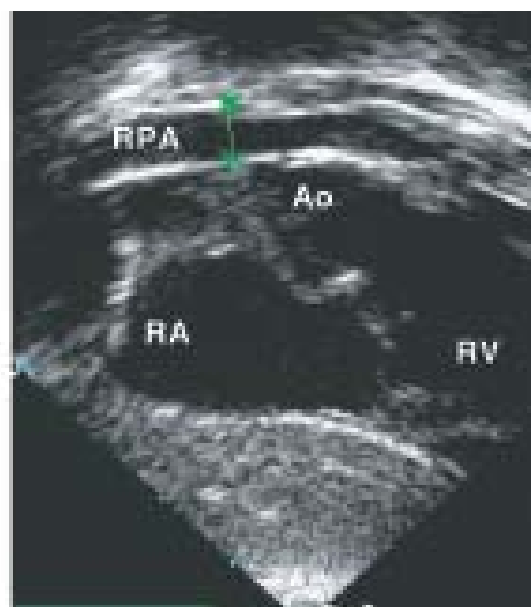

Fig - 5B

Fig.-5 (A,B) : Subcostal paracornal view axis view showing RPA.
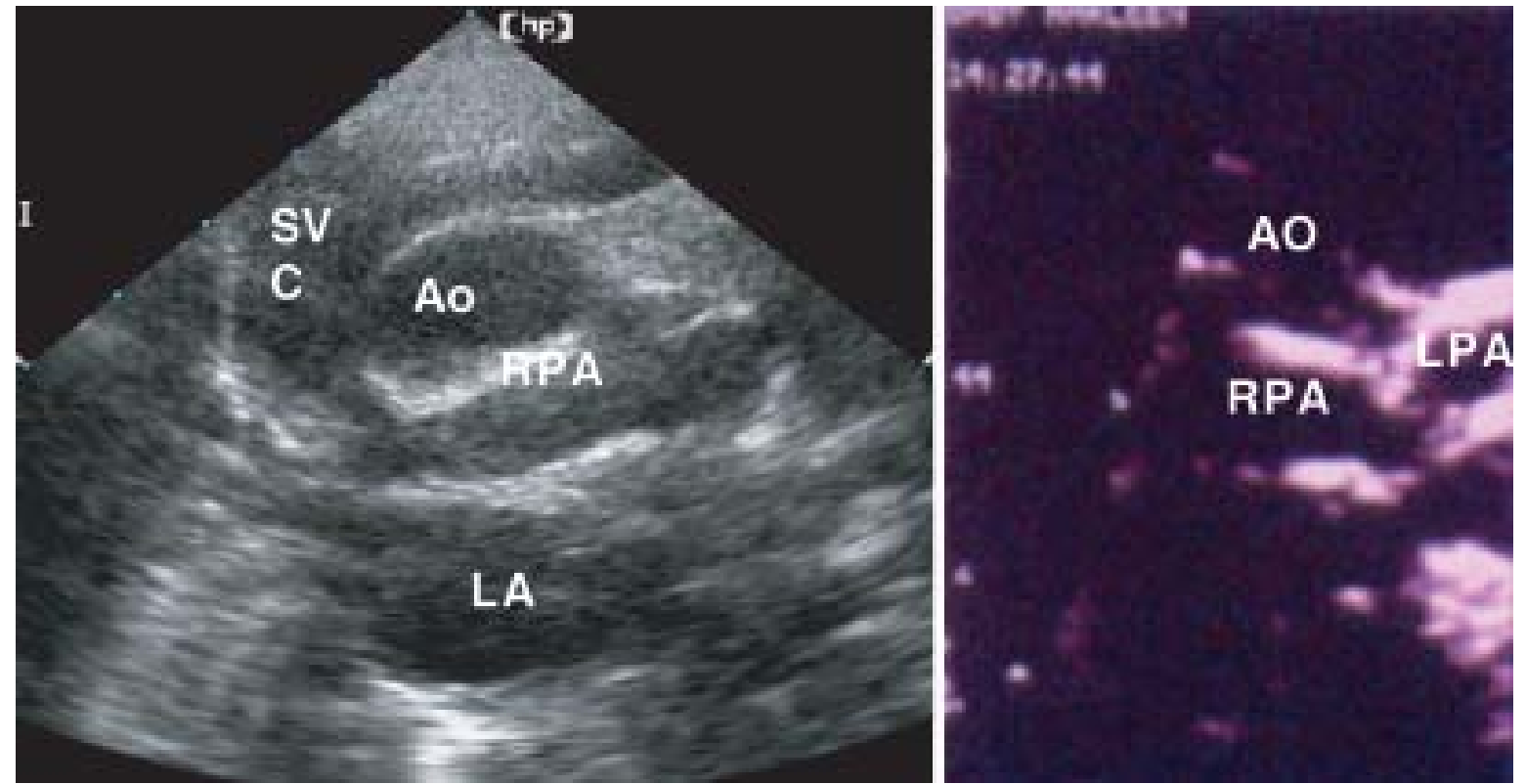

Fig.-6: Suprasternal short axis view showing confluent branch PA s.

\section{Aortic Arch-}

The suprasternal view is used to differentiate left (Fig7a, b) and right aortic arches (Fig 8a, b).

Left aortic arch - crosses left main stem bronchus

- passes left of Trachea (profiled in suprasternal and high left parasternal view)

- $1^{\text {st }}$ branch is right innominate which bifurcate into right subclavian and right carotid (profiled in supra sternal short axis view)

Right aortic arch - crosses right main stem bronchus

- passes right of Trachea (profiled in suprasternal view)

- $1^{\text {st }}$ branch is left innominate which bifurcate into left subclavian and left carotid (profiled in suprasternal short axis view) 

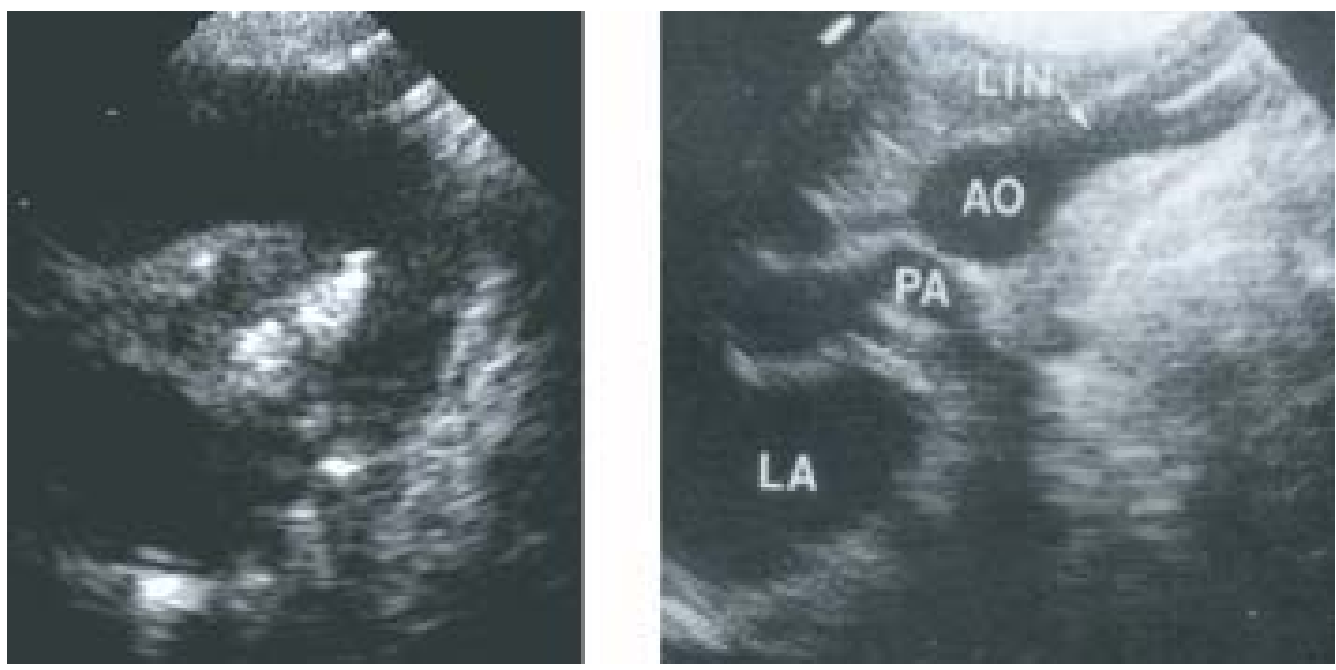

Fig.-7 (a,b) : Suprasternal long view axis view showing left aortic arch.
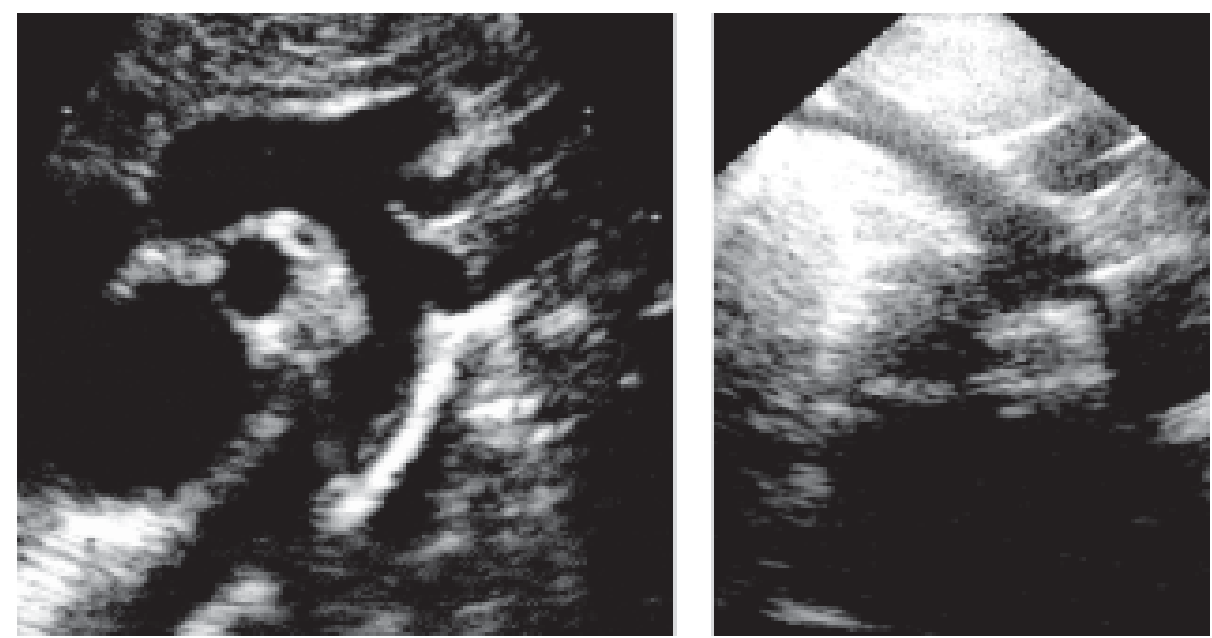

Fig.-8 (a,b) : Suprasternal long view axis view showing right aortic arch.

Patent arterial duct (PDA) and Aortopulmonary collaterals:

PDA is easily profiled with color Doppler flow as continuous flow in the left pulmonary artery at its insertion. Right-sided duct can rarely be seen in patients of tetralogy of Fallot. In such cases the continuous flow on color flow mapping is most prominent in the right pulmonary artery. Aortopulmonary collaterals are most often feature of tetralogy of Fallot with pulmonary atresia although they can also be present in patients with tetralogy of Fallot with pulmonary stenosis. The suprasternal views show the collateral arising from the descending aorta on color flow mapping. The presence of collaterals can also be suspected by profiling the descending thoracic aorta in its long axis from subcostal view (Fig. 9) while looking for collateral zoom mode with low colour scale is useful. It is usually not possible to further delineate the course of these collaterals in the lung parenchyma by echocardiography.

\section{Coronary artery anomalies:}

Normal coronary pattern is seen in $85-90 \%$ of the patients (Fig 10a, b). Surgically important coronary artery anomalies are seen in $3-15 \%$ of patients with tetralogy of Fallot. The parasternal short axis view is best to detect these anomalies. The most common anomalies seen are-1) origin of the left anterior descending artery (LAD) from the right 


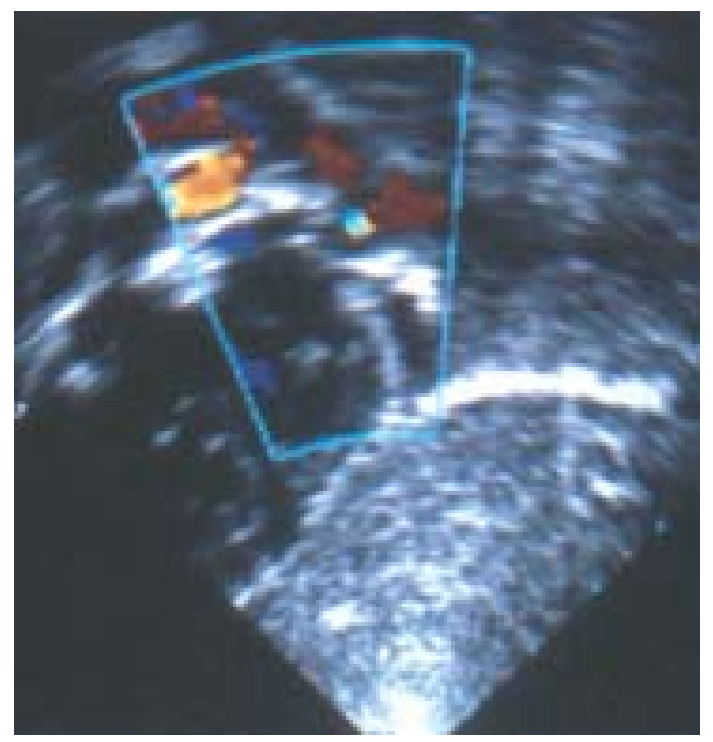

Fig.-9 : Subcoastal view showing collaterals from descending aorta.
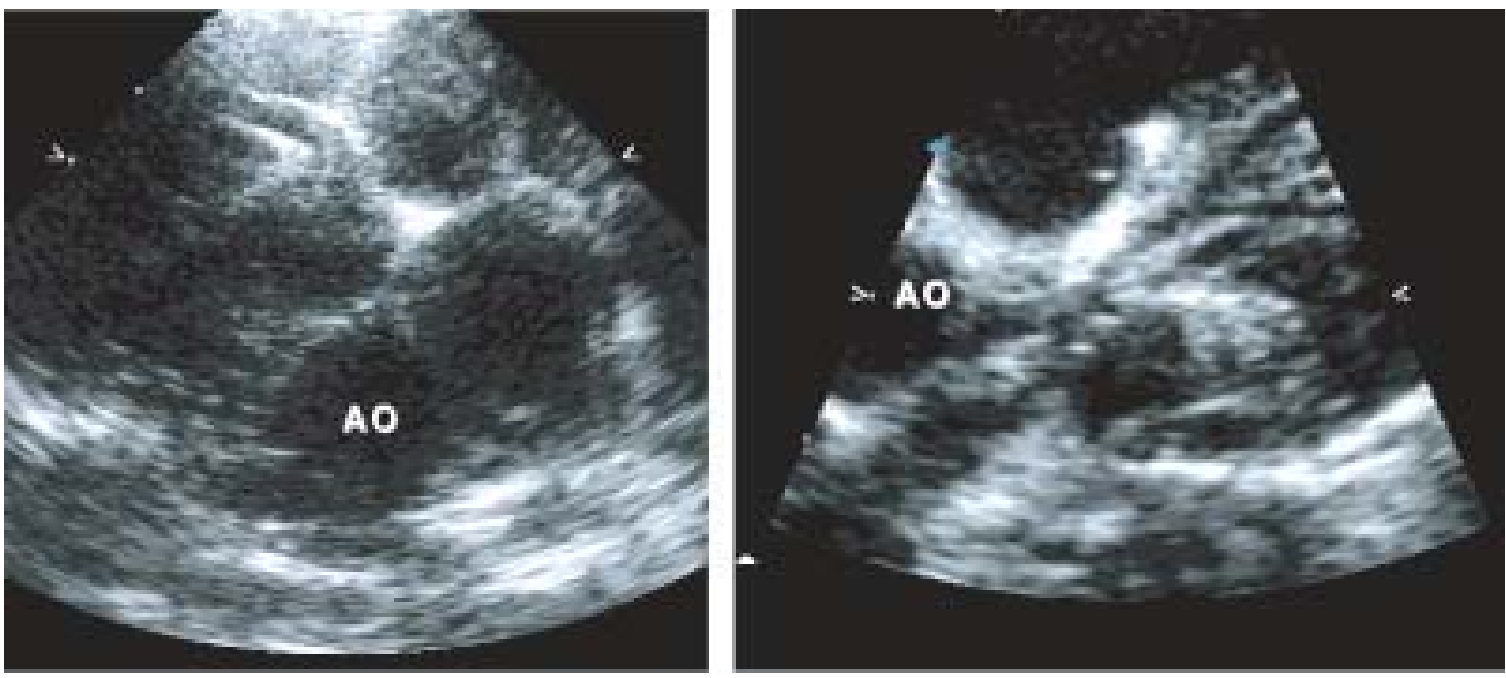

Fig.-10 (a,b) : Parastrenal short axis view showing normal coronary artery pattern in TOF.

coronary artery (RCA) .In the parasternal short axis view this is seen as a large branch arising from the right coronary artery and coursing left ward and anterior towards the right ventricular outflow tract and later the interventriculars septum (Fig. 11). 2) Single Right or Left Coronary artery 3) Dual LAD from right coronary artery and left coronary artery 4) Large Conal branch ${ }^{8,9}$ when the conal branch as large or bigger than RCA).

Echocardiographic Measurements in Tetralogy of Fallot-

The following measurements are routinely performed and compared with the nomogram based on weight of the patient. ${ }^{10,11}$
1. The pulmonary annulus: This is measured at the site of insertion of the pulmonary valve leaflet in parasternal short axis and right ventricular out flow view obtained by tilting the tranceducer posteriorinferorly and to the right in parasternal long axis plane (Fig. 4).

2. The right and left pulmonary artery at the hilum just before its first bifurcation: The suprasternal short axis view is selected for measuring the right pulmonary artery and the high parasternal or the oblique suprasternal long axis views for the left pulmonary artery. 


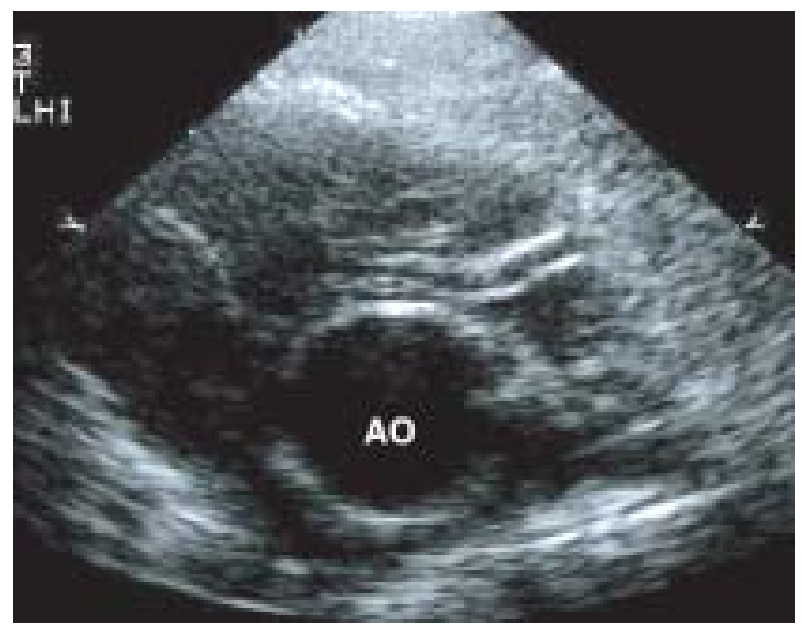

Fig.-11 : Parastrenal short axis view showing prominent $L A D$ from $R C A$.

Indications for cardiac catheterization in patients of tetralogy of Fallot-

Older children, adolescents and adults with tetralogy of Fallot may have suboptimal windows for echocardiography, so inability to delineate the above mentioned anatomic details is an indication for cardiac catheterization and angiocardiography /CT angio/MRI.

Cardiac catheterization may be needed if significant collaterals needing Coil closure before surgery. In TOF with pulmonary atresia many times adequate information is not obtained on Echocardiography and MRI / CT angio / cardiac

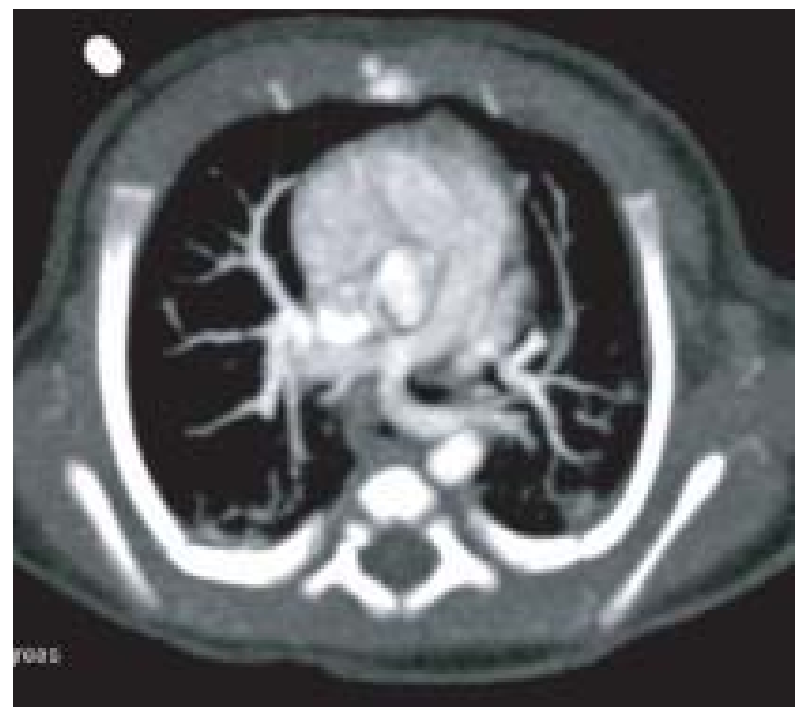

Fig.-12 : CT angio showing confluent branch pulmonary arteries in a case of TOF. catheterization and angiocardiography is needed. It is also sometimes indicated in evaluation of patients for complete repair of tetralogy of Fallot following shunt surgery for the following reasons.

1. To accurately delineate the pulmonary artery anatomy.

2. To determine pulmonary artery pressure. It is difficult to predict the pulmonary artery pressures accurately by Doppler evaluation even if the signals of patent shunt are good (due to the length of the shunt).

\section{Conclusions:}

Clinical assessment is essential for decision making. In most cases of TOF meticulously performed Echocardiographic study gives adequate information for surgical planning. In case of inadequate images on Echocardiography MRI/CT Angio may be needed. Cardiac Catheterization and angiocardiography are also essential for Coiling and in many cases of TOF pulmonary artesia before total correction.

\section{References:}

1. Ferencz C, Loffredo CA, Correa-Villasenor A, et al., eds. Malformations of the Cardiac Outflow Tract in Genetic and Environmental Risk Factors of Major Cardiovascular Malformations: The BaltimoreWashington Infant Study 1981-1989. Armonk, NY: Futura Publishing, 1997:59-102.

2. Emanuel BS, Budarf ML, Scambler PJ. The genetic basis of conotruncal cardiac defects: The chromosome 22q11.2 deletion. In: Harvey RP, Rosenthal N, eds. Heart Development. New York: Academic Press, 1999:463478.

3. Sanders SP, Bierman FZ, Williams RG, Conotruncal malformations diagnosis in infancy using subxiphoid two dimensional echocardiography. Am J Cardiol 1982;50:1361-1367.

4. Silove ED, De Giovanni JV, Shiu M F, Myint YI , Diagnosis of right ventricular outflow tract obstruction by cross sectional echocardiography. Br Heart $J$ 1983;50:416-420.

5. Capelli H , Somerville J , Atypical Fallots Tetrology with doubly committed subarterial VSD, Am J Cardiol 1983:51; 282-285.

6. Marino B, Bellarini L, Marcellitti C et al Right oblique subxiphoid view for two-dimensional echocardiographic 
visualization of the right ventricle in congenital heart disease. Am J Cardiol 1984 Nov 1;54 (8):1064-8.

7. Issaz K, Cloez JI, Marcon F et al .Is the aorta truly dexroposed in tetrology of Fallot ?. A two dimensional echocardiographic answer.Circulation 1986 ;73: 892899.

8. Juredini SB, Applton RS, Nouri S et al . Detection of coronary artery abnormalities in Tetrology of fallot by two dimesional echocardiography . J Am Coll of Cardiol 1989 : 14: 960 -967.

9. Need LR, Powell AJ, del Nido P, et al. Coronary echocardiography in tetralogy of Fallot: Diagnostic accuracy, resource utilization and surgical implications over 13 years. J Am Coll Cardiol 2000;36:1371-1377.

10. Flanagan MF, Foran RB, Van Praagh R et al . tetrology of fallot with obstruction of venstricular septal derfect spectrum of echocardiographic finding. $J \mathrm{Am}$ Coll Cardiol 1988;11:386-395.

11. Geva T, Ayres NA, Feyza PA, Pignatelli R. quantitative morphometric analysis of progressive infundibular obstruction in teratology of Fallot. A prospective longitudinal echocardiographic study. Circulation 1995:92(4): 886-892. 